\title{
The Rosette Eye: the key transition phase in the birth of a massive star
}

\author{
J. Z. Li \\ National Astronomical Observatories, Chinese Academy of Sciences, Beijing 100012, \\ China; ljz@bao.ac.cn \\ M. D. Smith \\ Centre for Astrophysics $\& 3$ Planetary Science, University of Kent, Canterbury CT2 7NH, \\ $U K$ \\ R. Gredel \\ Max-Planck Institut für Astronomie, Königstuhl 17, D-69117 Heidelberg, Germany \\ C. J. Davis \\ Joint Astronomy Centre, 660 North A'ohoku Place, Hilo, HI 96720 \\ T. A. Rector \\ University of Alaska at Anchorage, 3211 Providence Drive, Anchorage, AK 99508
}

\begin{abstract}
Massive protostars dramatically influence their surroundings via accretioninduced outflows and intense radiation fields. They evolve rapidly, the disk and infalling envelope being evaporated and dissipated in $\sim 10^{5}$ years. Consequently, they are very rare and investigating this important phase of early stellar evolution is extremely difficult. Here we present the discovery of a key transient phase in the emergence of a massive young star, in which ultraviolet radiation from the new-born giant has just punctured through its natal core. The massive young stellar object AFGL 961 II is readily resolved in the near infrared. Its morphology closely resembles a cat's eye and is here dubbed as the Rosette Eye. Emerging ionized flows blow out an hourglass shaped nebula, which, along with the existence of strong near-infrared excess, suggests the existence of an accretion disk in the perpendicular direction. The lobes of the hourglass, however, are capped with arcs of static $\mathrm{H}_{2}$ emission produced by fluorescence. This study has strong implications for our understanding of how massive stars embark on their formation.
\end{abstract}


Subject headings: stars: formation - stars: early type - stars: individual (AFGL 961 II) - accretion, accretion disks - ISM: jets and outflows

\section{Introduction}

Protostellar objects are, in most cases, deeply embedded in molecular clouds and are enshrouded by heavy foreground extinction. This makes the very early stages of stellar gestation notoriously illusive (Zinnecker \& Yorke 2007). The birth of stars with masses above $10 \mathrm{M}_{\odot}$ is particularly intriguing as their radiation is apparently sufficient to resist the accretion of gas and, hence, further mass growth unless somehow mitigated (Mckee \& Tan 2002; Behrend \& Maeder 2001). Evidence of potential disks and/or envelopes associated with massive star formation has been accumulated through various probes especially in the radio domain (Chini et al. 2004; Patel et al. 2005). However, the formation process of massive stars remains far from being resolved. The critical growth period of massive stars lasts only tens of thousand of years but is usually accompanied by spectacular ejections of gas in opposite directions. Since such jets are circumstantial evidence of an accretion disk, it is possible and crucial to gain robust evidence of ongoing accretion associated with massive young stellar objects (YSO) in their early stages of evolution.

The Rosette Molecular Complex (RMC) is a famous isolated massive star forming region with an extent of about 100 pc. It is located at a distance of $\sim 1.39 \mathrm{kpc}$ (Hensberge et al. 2000) and harbors a gas reservoir of $\sim 10^{5} M_{\odot}$ (Blitz \& Thaddeus 1980). New generation OB star formation is in evidence in the densest ridge of the Complex (Li \& Smith, 2005). The well known high-mass protostellar system AFGL 961 is situated well within this region (Cohen 1973; Grasdalen et al. 1983; Castelaz et al. 1985; Lenzen et al. 1984; Hodapp 1994). The third component of the system, designated as AFGL 961 II (Li \& Smith 2005), was first noticed to be associated with a small nebulosity by Eiroa (1981) and later briefly discussed by Hodapp (1994). The nebulosity was considered as a cavity with a partial shell surrounding the central young star (Aspin 1998; Alvarez et al. 2004). However, the origin of the intriguing YSO is far from clear.

\section{Observations and Data Reduction}

\subsection{Infrared imaging and spectroscopy}

We obtained near-infrared images of the AFGL 961 region as part of our program to explore the entire Rosette star formation complex. The JHK and $\mathrm{H}_{2}$ data were obtained 
on December 19-20, 2005, with the SOFI instrument (Moorwood, Cuby \& Lidman 1998) on the ESO New Technology Telescope at La Silla in Chile. The integration time is 2000s in the $\mathrm{H}_{2}$ 1-0 $\mathrm{S}(1)$ and 300s in each of the $\mathrm{J}, \mathrm{H}$ and $\mathrm{Ks}$ bands. We also employed SOFI to obtain broad Ks-band spectroscopy. The HR Grism at order 3 results in a medium spectral resolution of $\sim 2200$ over the wavelength range $2.00-2.30 \mu \mathrm{m}$. A $0^{\prime \prime} .6$ slit was used, cutting through both the exciting source and the bipolar shock structures at a position angle of $345^{\circ}$ (north to east). Four 300s exposures of the target source were taken, which was accompanied by a 300s exposure of the blank sky. A telluric standard star (Hip 1179 with a spectral type of A7V, Perryman et al. 1997) was observed right after the exposures of the target. The integration times are $3 \times 60 \mathrm{~s}$ for the standard star and $60 \mathrm{~s}$ for the sky background.

Near-infrared echelle spectra covering $\mathrm{H}_{2}$ 1-0 S(1) were obtained at the U.K. Infrared Telescope (UKIRT) on September 18, 2006 UT. The cooled grating spectrometer CGS 4 (Mountain et al. 1990) was used, which employs a $256 \times 256$ pixel InSb array and has a pixel scale of $0.41 " \times 0.88$ " (0.41" in the dispersion direction). A 2-pixel-wide slit was used which yields a velocity resolution of $\sim 16 \mathrm{~km} \mathrm{~s}^{-1}$. An internal black-body lamp was used to flat-field each spectral image, before the sky frames were subtracted from each object frame. The coadded spectral images were then wavelength calibrated using sky lines in the sky frame. The overall velocity calibration is accurate to better than $6 \mathrm{~km} \mathrm{~s}^{-1}$, while velocity shifts between adjacent spectra observed along the same slit are accurate to within $\sim 2 \mathrm{~km}$ $\mathrm{s}^{-1}$. Finally, observations of HD 42807 (BS 2208; G2V, V=6.22 mag) obtained immediately before the data were used to correct for telluric absorption and to flux calibrate each spectral image.

\subsection{Optical imaging}

The AFGL 961 system was observed on the night of 15 December 2006 with the Kitt Peak National Observatory 4-meter telescope and the Mosaic I camera (Muller et al. 1998). The pixel scale is $0.258^{\prime \prime}$ pixel $^{-1}$. Five 600 s exposures were obtained in each of the $\mathrm{H} \alpha(\mathrm{k} 1009)$ and $\mathrm{H} \alpha+16 \mathrm{~nm} /[\mathrm{SII}]$ (k1013) filters. The median seeing in both filters is about $0.9^{\prime \prime}$. The exposures were dithered so that the gaps between the CCDs were filled during the stacking process. The data were reduced with the IRAF MSCRED package in the standard manner. 


\subsection{Optical spectroscopy}

Medium resolution spectroscopy of AFGL 961 II was performed on the night of 22 January 2006 with the $2.16 \mathrm{~m}$ telescope of the National Astronomical Observatory of the Chinese Academy of Sciences (NAOC). An OMR (Optomechanics Rsearch Inc.) spectrograph and a Tecktronix 1024 x $1024 \mathrm{CCD}$ were used. A $50 \AA \mathrm{mm}^{-1}$ grating and a 2 " slit resulted in a two-pixel resolution of the spectra of $2.4 \AA$. The accuracy of the wavelength calibration allows sampling of velocities down to $20 \mathrm{~km} \mathrm{~s}^{-1}$.

\section{Results}

We resolve the nebulous YSO, AFGL 961 II, with our data into distinctive structures. Its spectacular appearance closely resembles a cat's eye in our NIR colour-composite image (Fig. 1) and we thus refer to it as the Rosette Eye. It is composed of a bright young star at the center, an hourglass shaped diffuse nebula oriented at a position angle of $345^{\circ}$ (from north to east), and extensive molecular emission resembling bipolar shock structures on either side of the apparent central source. The molecular emission is the most prominent in the welldefined arc-like structures of the system. A faint star is also found in close proximity to the central star (see Fig. 1), but does not appear to be physically related to the excitation of the extended nebulosity. The spatial appearance of the Eye, along with the detection of strong excesses in the near-infrared ( $\mathrm{Li} \&$ Smith, 2005), suggests the existence of an accretion disk in the perpendicular direction.

At a distance of $1.39 \mathrm{kpc}$ (Hensberge et al. 2000), the arc structures arch over an hourglass shaped cavity with a physical scale of $\sim 0.087 \mathrm{pc}$, which is in agreement with typical sizes of $\sim 0.1$ pc of massive star-forming clumps (Kurtz, et al. 2000; Motte \& Andre 2001). If we assume a molecular density of $\mathrm{n}_{H_{2}}=10^{7} \mathrm{~cm}^{-3}$ (De Pree et al. 1998, Motte et al. 2001, Churchwell 2002), a pre-stellar core of this size will harbor a gas reservoir of over $53.5 \mathrm{M}_{\odot}$ and is rich enough to give birth to a $20 \mathrm{M}_{\odot}$ ( $\mathrm{Li} \&$ Smith 2005 ) infant star according to theoretical models (Yorke \& Sonnhalter 2002).

The spatial appearance of the Rosette Eye in each of the observed bands is presented in Fig. 2, where we see a clear bipolar nebula in J, resembling an hourglass with an opening angle of about $60^{\circ}$. An eye structure is superimposed; the north-west arc is more distinctively

attached to the end of the conical nebula than is the south-east arc. There is also diffuse scattered light marginally visible beyond the north-west arc. The conical nebula to the southeast possesses a sharp edge, suggesting the existence of high extinction in that direction. Diffuse extensive emission extends further to the east, pointing toward the massive binary 
associated with AFGL 961, probably a leakage of the stellar light over the cavity walls. The hourglass-shaped nebula is less prominent in the $\mathrm{H}, \mathrm{Ks}$ and $\mathrm{H}_{2}$ bands with the apparent opening angle reduced to about $45^{\circ}$. The arc structure in the south-east, however, brightens remarkably as the wavelength increases. It is sharp and bright in the Ks band image and is most prominent in the $\mathrm{H}_{2}$ narrow-band image.

In the optical narrow-band $\mathrm{H} \alpha$, the structure to the north-west resembles that observed in the $\mathrm{J}$ band but displays more diffuse and extended emission. The south-east lobe appears to be dominated by heavy extinction and is restricted to the Eye structure. Our mid-resolution optical spectroscopy of the exciting source shows very strong $\mathrm{H} \alpha$ emission $\left(\mathrm{EW}=170 \AA, \mathrm{FWHM}=\sim 500 \mathrm{~km} \mathrm{~s}^{-1}\right)$. This, along with the broad $\operatorname{Br} \gamma$ emission presented below, is a good indicator of youth as also seen in other massive YSOs (Bunn et al. 1995). However, only marginal [SII] emission is detected from both the central YSO and the photoionized nebula. The spectroscopy with a resolution of $1.0 \AA$ pixel $^{-1}$ yields no velocity difference between the bipolar lobes. This suggests a low velocity of at most a few tens of $\mathrm{km} \mathrm{s}^{-1}$ of the outflowing gas, in accord with the general properties of outflows associated with massive protostellar objects (Churchwell 2002; Henning et al., 2000).

Given the optical spectroscopy results, the [SII] image closely represents continuum emission from the Eye. The derived continuum-subtracted $\mathrm{H} \alpha$ emission shows a small HII region excited by the UV ionizing photons from the YSO and faint net emission from the north-west arc, indicating marginal ionization in the shell. The small HII region is confined by the bipolar cavity, which is less than $0.1 \mathrm{pc}$ in size. Here the UV ionizing photons are attributed to shocked accretion from the proposed disk/envelope in the orthogonal direction, which feeds the YSO continuously till the final dissipation of the circumstellar materials. The morphology of the Eye matches well the transient phase in massive stellar evolution presented by Keto 2007, where an hourglass-shaped photon-dissipation region first forms before it evolves into a full-blown HII region. The excitation of the ionized nebula, however, suggests a spectral type earlier than B2 for the exciting star, commensurate with its position on the color-magnitude diagram in the NIR (Li \& Smith 2005).

Archived Spitzer IRAC and MIPS imaging data on the AFGL 961 region, which traces hot dust distribution, indicate that AFGL 961 II, the exciting source of the Eye, sits on the edge of a lane of dust with the highest extinction. The conical structure in the southeast encounters heavy dust extinction. This explains why the bipolar nebula is observed in the optical as a fan shaped nebula extending toward the north-west (Li \& Smith 2005). The MIPS $24 \mu \mathrm{m}$ imaging, on the other hand, indicates a dumbbell shaped nebula that encompasses the bipolar cavity detected in the NIR, which is devoid of dust emission and implies the dissipation or evacuation of dust by the ionized flows. With a moderate extinction 
of $\sim 7 \mathrm{mag}$ (Li \& Smith 2005) and a favorable viewing angle, we propose that AFGL 961 II represents a spectacular case of a massive star visible in its early stage of formation.

Strong $\mathrm{H}_{2}$ line emission often arises after collisional excitation within shock waves in association with mass ejections from extremely young stars. This usually yields relatively high radial velocities and strong excitation of the lower vibrational levels. To test this hypothesis, we performed deep Ks band spectroscopy with a slit position cutting through both the arc structures and the central YSO. The spectrum of the exciting source shows $\operatorname{Br} \gamma$ $(\mathrm{EW}=10.8 \AA, \mathrm{FWHM}=29 \AA)$ superimposed on a featureless rising continuum towards longer wavelengths. However, the $\mathrm{H}_{2}$ emission from the shell displays no sign of collisional excitation. Line emission originates from very high vibrational levels of $\mathrm{H}_{2}$ (Fig. 3, upper panel). The highest level present is the 9-7 Q(3) at $2.100 \mu \mathrm{m}$, originating $42,462 \mathrm{~K}$ above the ground state. The spectroscopic data demonstrate distinctly that the gas is purely fluoresced with the $\mathrm{H}_{2}$ line strengths corresponding exactly to an energy level cascade. This, however, is in agreement with theoretical predictions that UV pumped fluorescence should be a common phenomenon, especially on surfaces of molecular clumps illuminated by young massive stars (Gatley 1987). AFGL 961 II thus represents the first detection of pure fluorescent radiation directly associated with massive protostellar objects.

The fluorescence origin of the line emission is confirmed by our simulation based on the observed line flux ratios (Smith, Li \& Gredel et al. in prep.). Furthermore, the echelle spectrogram obtained by UKIRT discloses no radial velocity motion in the shell to the limits of our measurements $\left(\sim 6 \mathrm{~km} \mathrm{~s}^{-1}\right)$ (Fig. 3, lower panel).

\section{Summary \& discussion}

This study substantiates that the exciting source of the Rosette Eye, AFGL 961 II, has just finished its original collapse, that UV radiation from shocked massive accretion has recently turned on and that ionized stellar winds have begun to emerge in the polar directions. The shell left by the original collapse is now prepared to face the UV ionization from the newly born star. We suggest that the Eye may closely correspond to a transient phase immediately preceding that of the famous Orion OMC-1 outflow (Stone et al. 1995). In this scenario, the arcs will be subjected to fluid instabilities as an ensuing fast wind of low density emerges, which leads to the so-called fireworks as the shell gas is driven out in the form of dense bullets (McCaughrean \& Mac Low, 1997). The results presented in this paper corroborate the recent onset of the formation of a massive star in the ridge of the RMC, which is in a key transient phase of its emergence from the natal cloud and the development of the HII region. 
We are grateful to the referee, Robert Gehrz, for the many helpful comments and suggestions made. This work was supported by INTAS grant 4838 and funding from the National Natural Science Foundation of China through grant 10503006.

\section{REFERENCES}

Alvarez, C. \& Hoare, M. G., 2005, A\&A 440, 569

Alvarez, C., Hoare1, M., Glindemann, A., et al., 2004, A\&A 427, 505

Aspin, C., 1998, A\&A 335, 1040

Behrend, R., Maeder, A., 2001, Astron. Astrophys. 373, 190

Bunn, J. C., et al., 1995, MNRAS 272, 346

Castelaz, M. W., Grasdalen, G. L., Hackwell, J. A., et al., 1985, AJ 90, 1113

Chini R. et al., 2004, Nature 429, 155

Churchwell, E. 2002, Annu. Rev. Astron. Astrophys. 40, 27

Cohen, M., 1973, ApJ 185, L75

De Pree, C. G., Goss, W. M. \& Gaume, W. M. R. A., 1998, Astrophys. J. 500, 847

Eiroa, C., 1981, A\&AS 44, 77

Gatley, I. et al. 1987, ApJ 318, L73

Hensberge, H., Pavlovski, K. \& Verschueren, W., 2000, A\&A 358, 553

Henning, Th., Schreyer, K. \& Launhardt, R., et al., 2000, A\&A 353, 211

Hodapp, K. W., 1994, ApJS 94, 615

Keto, E., 2007, Astrophys. J. 666, 976

Kurtz, S., Cesaroni, R. \& Churchwell, E. et al., 2000, Protostars \& Planets IV, p299

Lada, C. J. 2001, Encycl. Astron. and Astrophys, Edited by Paul Murdin, article 1896

Lenzen, R., Hodapp, K. W., Reddmann, T., 1984, A\&A 137, 365 
Li, J. Z. \& Smith, M. D., 2005, AJ 130, 721

McCaughrean, M. J., Mac Low, M. W., 1997, Astron. J. 113, 391

McKee, C. F., Tan, J. C., 2002, Nature 416, 59

Moorwood, A., Cuby, J.G., Lidman, C., 1998, The Messenger 91, 9

Motte, F. \& Andre, P., 2001, A\&A 365, 440

Motte, F. \& Andre, P., Ward-Thompson, D., et al., 2001, A\&A 372, L41

Mountain, C. M., Robertson, D. J. \& Lee, T. J., et al., 1990, Proc. SPIE, 1235, 25

Muller, G. P., Reed, R., Armandroff, T., et al., 1998, Proc. SPIE, 3355, 577

Patel N. et al., 2005, Nature 437, 109

Perryman, M. A. C., 1997, A\&A 323, L49

Stone, J. M., Xu, J., Mundy, L. G., 1995, Nature 377, 315

Yorke, H. W., Sonnhalter, C, 2002, ApJ 569, 846

Zinnecker, H., Yorke, H. W., 2007, Annu. Rev. Astron. Astrophys. 45, 481 
Table 1: Line fluxes derived from the SOFI/NTT data for the south and north rims. Note that the 8-6 $\mathrm{O}(4)$ and 1-0 $\mathrm{S}(1)$ fluxes have been decomposed by using the CGS4/UKIRT data.

\begin{tabular}{lrrrlrrr}
\hline \hline Line & $\begin{array}{r}\text { wavelength } \\
(\mu \mathrm{m})\end{array}$ & $\begin{array}{r}\text { flux } \\
\text { South }\end{array}$ & $\begin{array}{r}\text { flux } \\
\text { North }\end{array}$ & Line & $\begin{array}{r}\text { wavelength } \\
(\mu \mathrm{m})\end{array}$ & $\begin{array}{r}\text { flux } \\
\text { South }\end{array}$ & $\begin{array}{r}\text { flux } \\
\text { North }\end{array}$ \\
\hline $1-0 \mathrm{~S}(2)$ & 2.033 & 38.60 & 20.70 & $4-3 \mathrm{~S}(6)$ & 2.146 & $<2.00$ & $<2.00$ \\
$8-6 \mathrm{O}(3)$ & 2.041 & 10.30 & 7.04 & $2-1 \mathrm{~S}(2)$ & 2.154 & 18.20 & 10.82 \\
$3-2 \mathrm{~S}(5)$ & 2.065 & 3.89 & 3.49 & $9-7 \mathrm{O}(2)$ & 2.172 & 3.36 & 3.12 \\
$12-9 \mathrm{O}(3)$ & 2.069 & $<3.00$ & $<3.00$ & $3-2 \mathrm{~S}(3)$ & 2.201 & 16.50 & 10.80 \\
$9-7 \mathrm{Q}(1)^{*}$ & 2.073 & & & $4-3 \mathrm{~S}(5)^{*}$ & 2.201 & & \\
$2-1 \mathrm{~S}(3)$ & 2.073 & 20.30 & 14.80 & $8-6 \mathrm{O}(5)$ & 2.210 & 9.91 & 6.76 \\
$9-7 \mathrm{Q}(2)$ & 2.084 & 3.09 & 3.36 & $1-0 \mathrm{~S}(0)$ & 2.223 & 69.40 & 32.50 \\
$9-7 \mathrm{Q}(3)$ & 2.100 & 5.59 & 4.39 & $2-1 \mathrm{~S}(1)$ & 2.247 & 43.70 & 30.40 \\
$7-5 \mathrm{O}(6)$ & 2.108 & $<3.5$ & $<3.5$ & $9-7 \mathrm{O}(3)$ & 2.253 & 10.20 & 6.17 \\
$8-6 \mathrm{O}(4)^{\dagger}$ & 2.121 & 10.35 & 5.00 & $4-3 \mathrm{~S}(4)$ & 2.268 & 4.95 & $<3.00$ \\
$1-0 \mathrm{~S}(1)^{\dagger}$ & 2.121 & 127.65 & 61.60 & $3-2 \mathrm{~S}(2)$ & 2.286 & 10.00 & 8.16 \\
$3-2 \mathrm{~S}(4)$ & 2.127 & 10.30 & 5.85 & & & & \\
\hline
\end{tabular}

Units of $10^{-19} \mathrm{~W} \mathrm{~m}^{-2}$. The $1 \sigma$ flux measurement uncertainty is $2 \times 10^{-19} \mathrm{~W} \mathrm{~m}^{-2}$ and 3.0 $\times 10^{-19} \mathrm{~W} \mathrm{~m}^{-2}$ beyond $2.25 \mu \mathrm{m}$. The $12-9 \mathrm{O}(3)$ flux upper limit is subject to considerable error due to the location near a strong line.

* - Line blended; no decomposition possible. $\dagger$ - relative line fluxes decomposed from echelle data in approximately the same regions. 


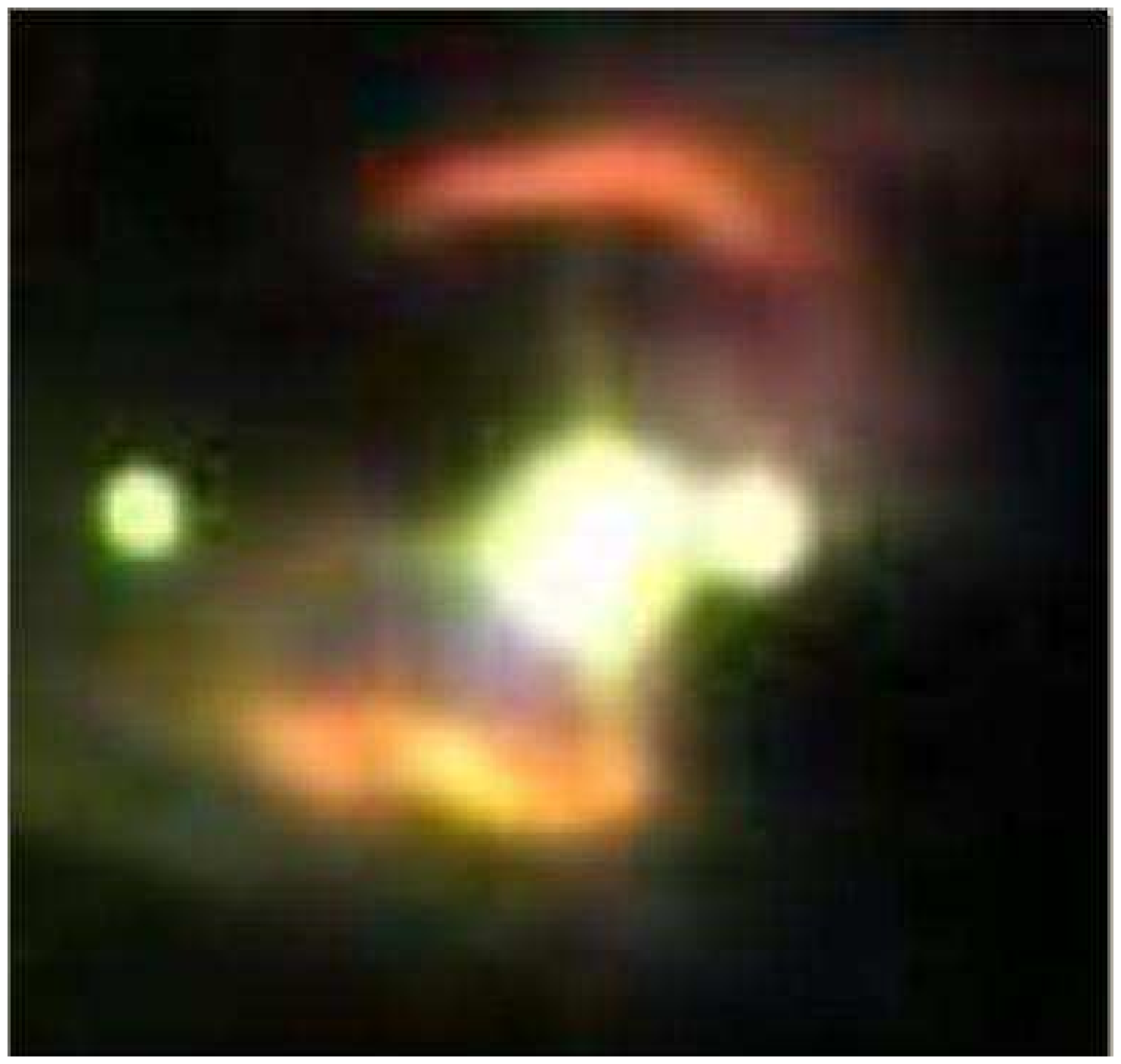

Fig. 1.- Close-up view of the Rosette Eye. North is up and East is to the left. The color composite with a size of $19^{\prime \prime} .0 \times 19^{\prime \prime} .0$ was compiled based on the NTT J (blue), H (green) and $\mathrm{H}_{2}$ (red) observations. The image displays both sharp outer features and inner diffuse emission. The northern rim is considerably redder and more distant from the central object. Note that the diffuse fan is stronger in the south with brighter outer edges, suggestive of a conical shell structure in three dimensions. 

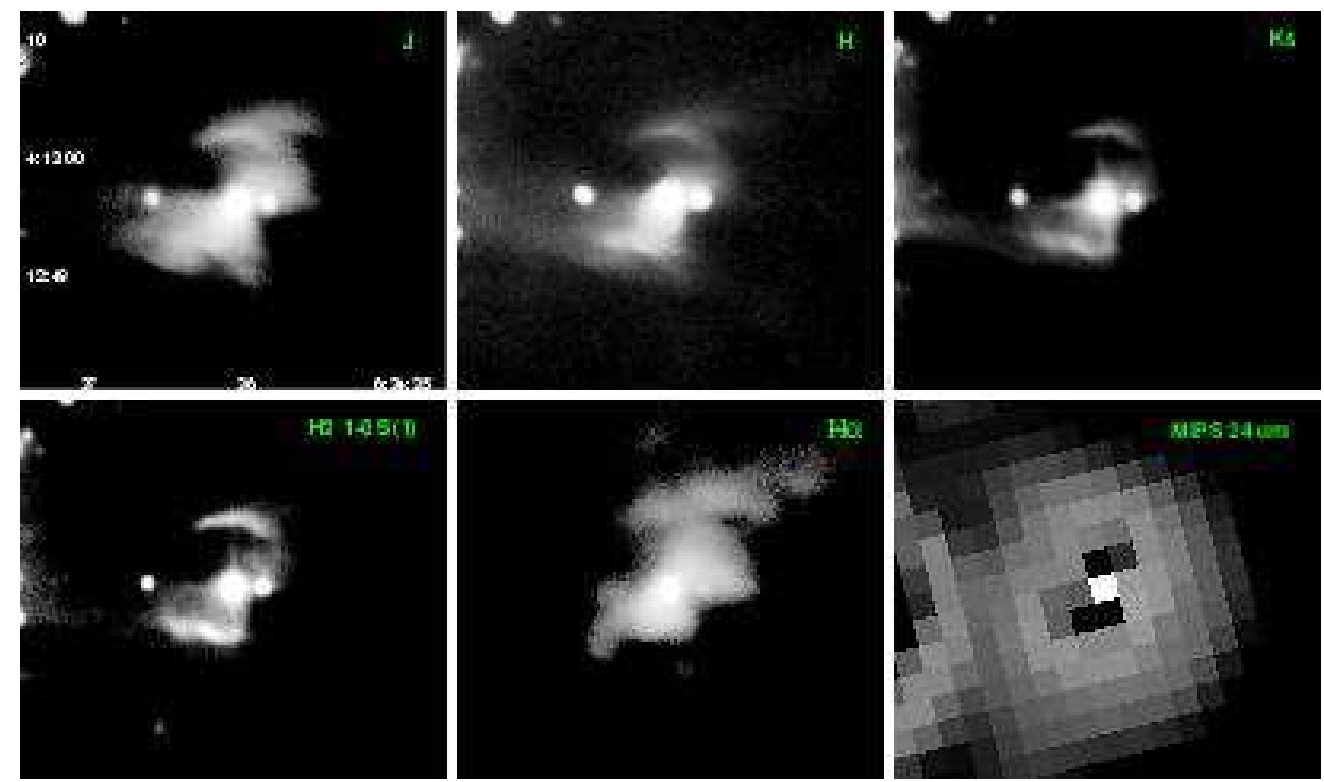

Fig. 2.- Greyscale images of the AFGL 961 II field in J (upper-left), H (upper-middle), Ks (upper-right), $2.12 \mu \mathrm{m}$ (lower-left), $\mathrm{H}_{\alpha}$ (lower-middle) \& MIPS $24 \mu \mathrm{m}$ (lower-right). North is up and East is to the left. The morphology of the Eye changes dramatically between bands. In the $\mathrm{J}$ band, it resembles an hourglass with an eye structure superimposed. In $\mathrm{H}$, $\mathrm{Ks}$ and $\mathrm{H}_{2}$, the Eye is prominent. In $\mathrm{H}_{\alpha}$, it indicates predominantly a fan shaped nebula that extends to the NW and higher extinction in the opposite direction. The outflow cavity is encompassed by a dumbbell shaped dusty bubble as disclosed by the MIPS $24 \mu \mathrm{m}$ image. 

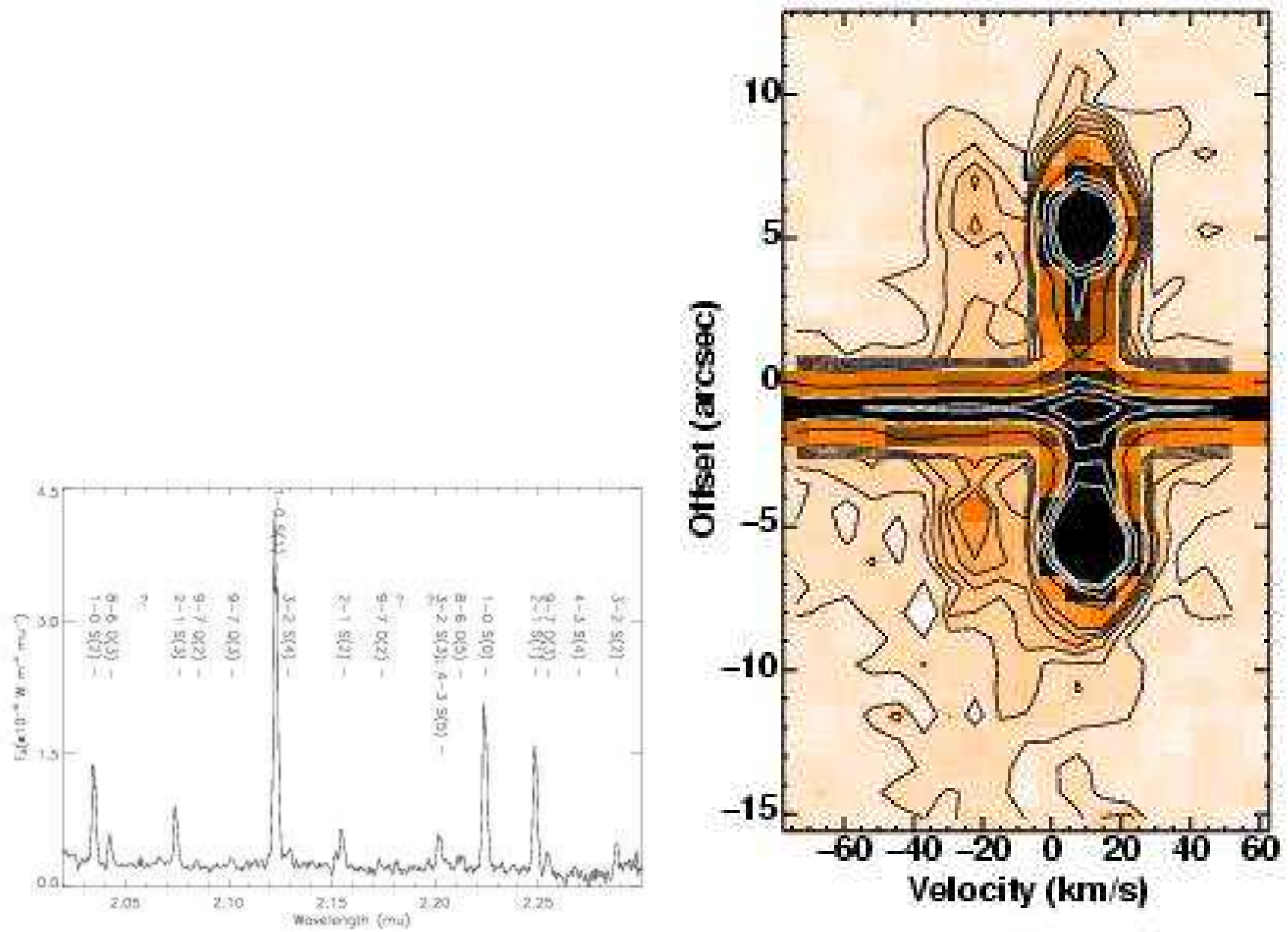

Fig. 3.- NTT Ks-band spectra of the arc structures (upper panel) with the identified molecular hydrogen lines indicated. It is obvious that line emission originating from very high vibrational levels of $H_{2}$ are present. The highest level present is the 9-7 Q(3) at $2.100 \mu \mathrm{m}$. It is thus conclusive that there is no indication of any shock emission at all. Instead, the sharp features are produced by an exemplary case of fluoresced $\mathrm{H}_{2}$. The PositionVelocity diagram of the 1-0 S(1) line emission from the Ks-band echelle spectroscopy obtained by UKIRT (lower panel) demonstrates that the material does not possess a measurable difference in radial velocity, as would be expected if it were shock-driven. Note that the echelle spectroscopy also uncovers one further high vibrational line, 3-2 S(4), that lies very close to the 1-0 S(1) line to its red side. 